\title{
Wissenschaftstheorie und Methoden
}

Joachim Behnke /Nathalie Behnke

\section{Die Bedeutung von Wissenschaftstheorie und Methoden}

Bevor wir mit unserer inhaltlichen Präsentation des „State of the Art" von Wissenschaftstheorie und Methoden in der deutschen Politikwissenschaft beginnen, möchten wir eine grundlegende Vorbemerkung machen. Auch wenn der Trend zur Internationalisierung der Forschung ein allgemeiner ist und für alle Fachgebiet gilt, so scheint uns dies für die Methoden in ganz besonderer Weise zuzutreffen. Für eine Wissenschaftsgemeinde, von der sich ein immer größerer Anteil in Hinsicht auf Forschungs- und Publikationstätigkeit im internationalen Umfeld bewegt, ${ }^{1}$ ist es selbstverständlich, dass sie den international gebräuchlichen Standards zu genügen versteht. Ein wesentlicher Teil der Entwicklung des Stands der Forschung auf diesem Gebiet spiegelt daher schlicht die internationale Entwicklung wider, mit einem starken Fokus auf der Entwicklung in den USA, d.h. den dortigen Zeitschriften, die unbestreitbar die Maßstäbe bestimmen. Eigenständige Beiträge deutscher Forscher, soweit es sie gibt, und es gibt sie durchaus, ${ }^{2}$ fließen dann in diesen internationalen, amerikanisch dominierten Schmelztiegel der einzelnen nationalen Entwicklungen der Forschungsmethoden mit ein. In jedem Fall ist die Entwicklung der Methoden jedoch derart vielfältig und umfangreich, dass wir in diesem Artikel auf keinen Fall allen Entwicklungen gerecht werden und sie mit gebührender Breite behandeln können. Lücken der Darstellung sind also sowohl dem Umfang des geschilderten Gegenstands geschuldet, als auch den Schwerpunkten im verfügbaren Wissen zum Gegenstand der Autoren selbst.

Die Frage, was denn gute von schlechter Wissenschaft unterscheidet, mündet in der Regel in die grundlegendere Frage, was denn überhaupt den „wissenschaftlichen" Charakter der Wissenschaft ausmacht, die wiederum als die Kernfrage der Wissenschaftstheorie bezeichnet werden kann. Die Beantwortung dieser Frage kann in zwei Komponenten zerlegt werden: Zum einen, was denn die

Vgl. Thomas Plümper, Publikationstätigkeit und Rezeptionserfolg der deutschen Politikwissenschaft in internationalen Fachzeitschriften, 1990-2002. Eine bibliometrische Analyse der Veröffentlichungsleistung deutscher politikwissenschaftlicher Fachbereiche und Institute, in: Politische Vierteljahresschrift, 44 (2003), S. 529-544.

2 Bernhard Kittel, Eine Disziplin auf der Suche nach Wissenschaftlichkeit: Entwicklung und Stand der Methoden in der deutschen Politikwissenschaft, in: Politische Vierteljahresschrift, 50 (2009), S. 577-603. 
Mindesterfordernisse sind, denen eine Arbeit genügen muss, damit sie mit Recht wissenschaftlich genannt werden darf, und zum anderen, darauf aufbauend, welche Regeln beim Vorgehen des Wissenschaftlers die Einhaltung dieser Mindesterfordernisse zu gewährleisten helfen.

Das wichtigste dieser Mindesterfordernisse ist das der Objektivität, oder vielleicht besser ausgedrückt -, das eines „objektiven“ Kerns jeder wissenschaftlichen Erkenntnis. Ohne die Annahme „objektiv“ richtiger Aussagen kann es keinen Erkenntnisfortschritt geben, da sonst die Erkenntnisse des einen die Irrtümer und Widerlegungen des anderen wären. Damit soll nicht gesagt werden, dass wir die Wirklichkeit „objektiv“" so wahrnehmen, wie sie tatsächlich ist, denn dass dies nicht der Fall ist, kann wohl inzwischen als erkenntnistheoretischer Allgemeinplatz angesehen werden. Objektiv meint vielmehr lediglich, dass es uns möglich ist, uns intersubjektiv nach bestimmten Kriterien darauf zu einigen, bestimmte Aussagen als wahr bzw. als falsch anzuerkennen oder zumindest als angemessene oder unangemessene Beschreibungen der Wirklichkeit zu betrachten. Regeln und Standards der Wissenschaftlichkeit sollen diese Kriterien im Einzelnen festlegen. Eine Aussage, die nach diesen Regeln gewonnen wurde und damit den Standards der Wissenschaftlichkeit genügt, sollte daher als glaubwürdiger angesehen werden als eine Aussage, die elementare Standards der Wissenschaftlichkeit verletzt. Methoden können nun spezifische Vorgehensweisen genannt werden, die die Einhaltung dieser Regeln und Standards gewährleisten. Zwar ist die Voraussetzung für die empirische Erforschung der Wirklichkeit die Verständigung darüber, dass es nur eine Wirklichkeit gibt; aber es gibt verschiedene Möglichkeiten (Methoden), sich dieser Wirklichkeit zu nähern. Daraus resultieren verschiedene Möglichkeiten, die Wirklichkeit zu beschreiben. Diese unterschiedlichen Wirklichkeitsbeschreibungen können sich entsprechend ihrer Akzentsetzung ergänzen oder gar komplementär zueinander verhalten, aber es kann nicht möglich sein, dass es zwei valide Wirklichkeitsbeschreibungen gibt, die gänzlich unvereinbar miteinander sind.

Jede Methode dient in diesem Sinne als eine Art von Entdeckungsverfahren von Gründen, warum man bestimmte Aussagen als gute und nützliche Beschreibungen der Wirklichkeit ansehen sollte. Die Pluralität der Herangehensweisen widerspricht nicht dem unitarischen Wirklichkeitsbild, sondern dient vielmehr dazu, dieses mosaikhaft zusammenzusetzen. Im Hinblick auf den Gegenstandsbereich der politikwissenschaftlichen Forschung - die Wirkung politischer Institutionen, das Handeln und die Wechselwirkung von Handlungen politischer Akteure, die Verortung von Macht - wird offensichtlich, wie unterschiedlich diese Wirklichkeitsausschnitte wahrgenommen und interpretiert werden können.
So fundamental das Nachdenken über wissenschafts- und erkenntnistheoretische Grundlagen für die Anwendung empirischer Methoden in der Forschung sein mag, so muss man doch konstatieren, dass die Wissenschaftstheorie sowohl in der institutionalisierten Methodenforschung als auch in den einschlägigen Methodenlehrbüchern eher ein Schattendasein fristet.

Ein institutionalisierter Zusammenschluss methodisch orientierter Politikwissenschaftlerinnen und Politikwissenschaftler erfolgte in Deutschland erst 2003 mit der Gründung der Ad-hoc Gruppe (später Arbeitskreis) "Empirische Methoden der Politikwissenschaft" in der Deutschen Vereinigung für Politische Wissenschaften (DVPW). Die politikwissenschaftliche deutsche Methodendebatte ist wiederum stark geprägt durch die institutionalisierte Methodenforschung in der Deutschen Gesellschaft für Soziologie (DGS) einerseits und der American Political Science Association (APSA) andererseits. Beide Vereinigungen verfügen seit langem über mitgliederstarke Methodensektionen, in denen die methodischen Entwicklungen vorangetrieben wurden, die von der deutschen Politikwissenschaft im Wesentlichen rezipiert wurden.

In den einschlägigen Lehrbüchern der Disziplin finden wissenschaftstheoretische Ausführungen nur wenig Raum. Anhänger der quantitativen Forschungstradition begründen die Wissenschaftlichkeit ihres Vorgehens in der Regel durch die Einhaltung bestimmter methodischer Standards, wofür das vermutlich einflussreichste Buch zur Methodologie der letzten Jahrzehnte, „Designing Social Inquiry" von Gary King, Robert Keohane und Sidney Verba, ${ }^{3}$ beispielhaft steht. Herausragende Lehrbücher, die die spezifischen, aber nichtsdestoweniger allgemeingültigen Anforderungen an Wissenschaftlichkeit für die Sozialwissenschaften und die verschiedenen Wege, diese zu erfüllen, aus einer grundlegenden philosophischen Perspektive darstellen, wie z.B. Hollis, ${ }^{4}$ sind auf dem deutschsprachigen Markt nicht zu finden. Soweit Wissenschaftstheorie erwähnt wird, findet sie sich vorwiegend in Lehrbüchern zur Methodologie oder Methoden der Sozialwissenschaften allgemein. Für ersteres steht im deutschsprachigen Raum wohl immer noch paradigmatisch Karl-Dieter Opps „Methodologie der Sozialwissenschaften", die 2005 in sechster Auflage erschienen ist, für letztere Einführungen in die Methoden der Politikwissenschaft wie die von Dreier, ${ }^{5}$ Behnke, Baur und Behnke $^{6}$ und Westle ${ }^{7}$. Dabei ist die Orientierung an den drei großen modernen Klassikern der Wissenschaftstheorie, Carnap, Hempel und Popper, vorherr-

3 Gary King/Robert O. Keohane/Sidney Verba, Designing Social Inquiry, Princeton, New Jersey 1994.

4 Martin Hollis, The Philosophy of Social Science, Cambridge 1994.

Volker Dreier, Empirische Politikforschung, München 1997.

6 Joachim Behnke/Nina Baur/ Nathalie Behnke, Empirische Methoden der Politikwissenschaft, Paderborn 2010.

Bettina Westle (Hrsg.), Methoden der Politikwissenschaft, Baden-Baden 2009. 
schend. Vom empirisch-analytischen Paradigma abweichende oder dieses kritische rezipierende erkenntnistheoretische Überlegungen werden vor allem in Lehrbüchern der qualitativen Forschung angestellt. Hier ist einerseits das einflussreiche Buch zur Grounded Theory von Glaser und Strauss ${ }^{8}$ zu nennen, das als moderner Klassiker der qualitativen Methodologie bezeichnet werden kann, oder für die neuere Zeit der Sammelband von Brady und Collier, ${ }^{9}$ der eine Reihe von Beiträgen beinhaltet, die sich kritisch mit „Designing Social Inquiry“ auseinandersetzen. Generell ist der Hang zur Diskussion von erkenntnistheoretischen Grundsatzfragen im qualitativen Lager ausgeprägter, was sich stellvertretend an diesem Buch festmachen lässt. Für den deutschen Markt sei hier das Einführungsbuch von Blatter, Janning und Wagemann genannt. ${ }^{10}$

\section{Entwicklungstrends}

Für die methodische Entwicklung der Politikwissenschaft in den vergangenen 25 Jahren lassen sich vor allem zwei einflussreiche Trends ausmachen. Zum einen gilt, ähnlich wie es auch für die Soziologie konstatiert wurde, ${ }^{11}$ eine nachhaltige Veränderung durch die rasante Entwicklung immer leistungsstärkerer Computer und die Einführung des Internet. Zum anderen lässt sich eine deutliche methodische Professionalisierung der Disziplin in Lehre und Forschung konstatieren. ${ }^{12}$

Die flächendeckende Verbreitung leistungsstarker Computer hat für die Verfügbarkeit und Verarbeitbarkeit von Daten eine nachgerade revolutionäre Bedeutung gehabt. Im Hinblick auf die statistische Analyse von Daten bedeutete dies, dass nahezu beliebig große Datensätze in komplexen Analysen binnen kürzester Zeit ausgewertet werden können. Bspw. Pfadanalysen oder Programmschleifen die früher an Großrechnern noch tagelang gerechnet wurden, können heute an PC's in wenigen Minuten erstellt werden. Auch die in jüngerer Zeit größere Verbreitung bayesianischer statistischer Verfahren lässt sich wohl zum großen Teil mit den neuen Rechenkapazitäten erklären, denn die hierzu notwendigen aufwändigen Berechnungen hätten noch vor wenigen Jahren die PCs regelrech lahmgelegt.

8 Barney G. Glaser/Anselm L. Strauss, The Discovery of Grounded Theory, New York, N.Y. 1967.

9 Henry E. Brady/David Collier (Hrsg.), Rethinking Social Inquiry: Diverse Tools, Shared Standards, Lanham 2004

10 Vgl. Joachim K. Blatter/Frank Janning/Claudius Wagemann, Qualitative Politikanalyse. Eine Einführung in Forschungsansätze und Methoden, Wiesbaden 2007.

11 Vgl. Andreas Diekmann, Aktuelle Probleme der empirischen Sozialforschung, in: Andreas Diekmann (Hrsg.), Methoden der Sozialforschung, Wiesbaden 2006, S. 8-32.

12 Vgl. B. Kittel (Anm.2), S. 577-603.
Bei der Software zeichnet sich noch eine andere grundlegende Entwicklung $\mathrm{ab}$, die sich insbesondere bei den statistischen Programmpaketen aufzeigen lässt. Während PASW (früher SPSS) z.B. in der kommerziellen Meinungsforschung immer noch mehr oder weniger dominierend ist, ist es innerhalb der akademisch orientierten Forschergemeinde von Stata und inzwischen auch $\mathrm{R}$ abgelöst worden. Die bei Stata teilweise und bei R gänzlich umgesetzte Open-SourceIdeologie, verknüpft mit den im Vergleich zu SPSS deutlich weiter entwickelten Möglichkeiten der beiden Softwarepakete zur Programmierung eigener Prozeduren, hat dazu geführt, dass neueste methodische Entwicklungen im Softwarebereich schneller Eingang in die wissenschaftliche Auswertungspraxis finden. Aber auch ansonsten ist ein Trend zu erkennen, dass immer mehr Software, in der Regel zur Lösung bestimmter Probleme oder der Behandlung spezifischer Datenstrukturen, nicht mehr kommerziell sondern umsonst angeboten wird. Stellvertretend sei nur auf das kostenlos herunterladbare Softwareangebot auf der Website von Gary King verwiesen, ${ }^{13}$ auf dem sich u.a. die Software Amelia für multiple Imputation fehlender Daten oder das Programm EI zur Durchführung ökologischer Regressionen befinden. Doch die schöne neue Welt der kostenlosen Softwareangebote ist nicht nur auf Statistikprogramme beschränkt, kostenlos verfügbar sind u.a. auch Programme zur agentenbasierten Modellierung wie NetLogo (http://ccl.northwestern.edu/netlogo/) oder Repast (http://repast.Source forge.net/index.html) oder zur Bestimmung von Positionen auf Dimensionen mit Hilfe des Programms Wordscores (http://www.ted.ie /Political Science/word scores/), das in Stata eingebunden werden kann und demnächst auch als standalone-Lösung in Java zur Verfügung stehen wird. Auch wenn hier insgesamt die überseeischen Angebote noch eindeutig dominieren, so gibt es inzwischen auch aus dem deutschen Raum entwickelte kostenlose Softwareangebote, bspw. das Programm KOALA zur Untersuchung von Koalitionsbildungsprozessen, ${ }^{14}$ das spezifische Interessen von Politikwissenschaftlern anspricht, oder die von Martin Elff entwickelten R-Packages memisc und munfold, letzteres ein Programm, das eine multidimensionale Entfaltung ermöglicht und u.a. zur Verarbeitung von Daten des Comparative Manifesto Projects geeignet ist.

3 Vgl. Gary King, http//gking harvard.edu/stats.shtml.

14 Susumu Shikg te Entscheidungsmodelle in der Koalitionsforschung, in: Kai-Uwe Schnapp/Nathalie Behnke/Joachim Behnke (Hrsg.), Datenwelten. Datenerhebung und Datenbestände in der Politikwissenschaft, Baden-Baden 2009, S. 284-293. 
Auch für die qualitative Forschung hat die Entwicklung von Software (Computer Assisted Qualitative Data Analysis Software - CAQDAS) ${ }^{15}$ die systematische Anwendung komplexer Auswertungsstrategien - namentlich in der Tradition der "Grounded Theory" - in ganz anderem Umfang und Verbreitungsgrad ermöglicht und zugleich dazu beigetragen, dass sich auch für qualitative Auswertungsstrategien gewisse Standards und Qualitätsmerkmale etabliert haben. ${ }^{16}$

Bestanden noch Anfang der 1990er-Jahre große Forschungsprojekte vornehmlich in der Verfügbar- und Vergleichbarmachung von Datensätzen für die Sekundäranalyse, so hat die Möglichkeit der elektronischen Archivierung von Daten zum systematischen Aufbau nationaler und internationaler Datenarchive geführt. ${ }^{17}$ Für den deutschsprachigen Raum von herausragender Bedeutung ist das Datenarchiv für Sozialforschung der GESIS (www.gesis.org). Dort sind im Wesentlichen Umfragedaten bis in die 1950er-Jahre hin elektronisch verfügbar und teilweise im Volltext nach Frageformulierungen durchsuchbar. Durch die leichte Zugänglichkeit riesiger Mengen an Daten wurden die Möglichkeiten systematischer Sekundärdatenanalyse wesentlich erweitert. Dazu kommt noch, dass nicht nur forschungsproduzierte Daten nun in großem Umfang für Sekundäranalysen elektronisch zur Verfügung stehen; auch prozessproduzierte Daten sind über das Internet in großer Menge per Mausklick verfügbar. Man denke bspw. nur an die Archive parlamentarischer Debatten und Drucksachen, die von den Parlamenten auch rückwirkend immer weiter öffentlich zur Verfügung gestellt werden; die Informationen der statistischen Ämter, die anonymisierte 'public use'-files zum Download anbieten; oder die riesige Menge an Dokumenten und Informationen, die von der Europäischen Kommission im Internet verfügbar gemacht wird. Der Umfang und die Vielfalt der verfügbaren Datenquellen machen die empirische Erforschung politischer Sachverhalte teilweise überhaupt erst möglich.

Zeitlich einhergehend mit und wohl auch teilweise bedingt durch die explosionsartige Verbreiterung der Verfügbarkeit von Daten und ihrer Analysemöglichkeiten lässt sich ein deutlicher methodischer Professionalisierungsschub der Politikwissenschaft konstatieren, und zwar sowohl in der Forschung und Publikationspraxis als auch in der Lehre. War die Methodenausbildung in den politikwissenschaftlichen Studiengängen noch vor 15 bis 20 Jahren zumeist auf 2 SWS '-

15 Diese Computerprogramme, z.B. AtlasTi, das für den us-amerikanischen Markt entwickelt wurde, oder das deutsche Konkurrenzprodukt WinMax, bieten Tools, die genau auf die methodische Vorgehensweise der Grounded Theory abgestimmt sind, und die Übersicht über die komplexen Datenstrukturen wesentlich erleichtern.

16 B. G. Glaser/A. L. Strauss (Anm. 8); Udo Kuckartz, Einführung in die computergestützte Analyse qualitativer Daten, Wiesbaden 2009.

17 N. Behnke, Datenarchive: Überblick und Kommentierung, in: Kai-Uwe Schnapp/N Behnke/J. Behnke (Hrsg.), Datenwelten. Datenerhebung und Datenbestände in der Politikwissenschaft, Baden-Baden 2009, S. 392-408.
Fremdleistung' aus den Nachbardisziplinen beschränkt, so sind in den neu eingeführten BA-Studiengängen mittlerweile 6-8 SWS mit einem ausdifferenzierten Curriculum (i.d.R. Einführung in die Methoden, deskriptive und schließende Statistik, teilweise ergänzt durch Forschungspraktika, Wissenschaftstheorie, qualitative Methoden oder Vertiefungsveranstaltungen) die Regel.

Dass die Methodenausbildung nun im Rahmen der politikwissenschaftlichen Lehre selbst in die Hand genommen wurde, zeigt sich zum einen an der deutlichen Zunahme politikwissenschaftlich denominierter Lehrstühle mit einem Schwerpunkt in der Methodenausbildung, zum anderen an dem stetig wachsenden Angebot an Lehrbüchern: Der Sammelband von Behnke, Gschwend, Schindler und Schnapp stellt annähernd 30 neuere Analyseverfahren vor, die in den letzten Jahren Eingang in den Methodenkanon der deutschen Politikwissenschaf gefunden haben. ${ }^{18}$ Mit einem zweiten Sammelband von Schnapp, Behnke und Behnke, ${ }^{19}$ der ebenfalls aus dem Umfeld des DVPW-Arbeitskreises „Empirische Methoden der Politikwissenschaft“ entstanden ist, sollte der Fokus der Methodenanwendung stärker auf den Bereich der Gewinnung von Daten als auf deren Auswertung gerichtet werden. Auf das Design einer empirischen Forschungsarbeit geht der Band von Gschwend und Schimmelfennig ein, ${ }^{20}$ wobei hier vor allem Fragen in Hinsicht auf das Design eines Untersuchung ${ }^{21}$ sprojekts im Rahmen einer wissenschaftlichen Qualifikationsarbeit angesprochen werden.

Auch im engeren Bereich von Erhebungs- und Auswertungsmethoden hat sich in den letzten Jahren ein beachtliches Angebot an Publikationen ergeben, die sich spezifisch an den Bedürfnissen von Politologen ausrichten. Im Bereich der vergleichenden Methoden sind mehrere Sammelbände erschienen, die methodisch reflektierte Beiträge enthalten, so der von Kropp und Minkenberg oder der von Pickel, Pickel, Lauth und Jahn, ${ }^{22}$ sowie einführende Lehrbücher wie das von Lauth, Pickel und Pickel. ${ }^{23}$ Wenngleich es keinen disziplinabhängigen Unterschied der statistischen Methoden gibt, hat sich auch hier ein gewisser Lehrbuchmarkt entwickelt, der den Studierenden der Politikwissenschaft vor allem

18 Kai-Uwe Schnapp/N. Behnke/J. Behnke (Hrsg.), Datenwelten. Datenerhebung und Datenbestände in der Politikwissenschaft, Baden-Baden 2009.

19 J. Behnke/Thomas Gschwend/Delia Schindler/K.-U. Schnapp (Hrsg.), Methoden der Politikwissenschaft. Neuere qualitative und quantitative Analyseverfahren, Baden-Baden 2006 .

20 Thomas Gschwend/Frank Schimmelfennig (Hrsg.), Forschungsdesign in der Politikwissenschaft: Probleme - Strategien - Anwendungen, Frankfurt am Main 2007.

21 Sabine Kropp/Michael Minkenberg (Hrsg.), Vergleichen in der Politikwissenschaft, Wiesbaden 2005

22 Susanne Pickel/Gert Pickel/Hans-Joachim Lauth/Detlef Jahn (Hrsg.), Methoden der vergleichenden Politik- und Sozialwissenschaft. Neue Entwicklungen und Anwendungen, Wiesbaden 2009

23 Hans-Joachim Lauth/Gert Pickel/Susanne Pickel (Hrsg.), Methoden der vergleichenden Politikwissenschaft. Eine Einführung, Wiesbaden 2009. 
durch Anwendungen aus ihrem Gebiet entgegenzukommen versucht. Gehring und Weins führen auf didaktisch gelungene Weise in die Grundlagen der Auswertung von Umfragedaten ein. ${ }^{24}$ Behnke und Behnke sind ebenfalls schwerpunktmäßig auf Umfragedaten hin ausgerichtet, ${ }^{25}$ das Buch enthält zudem einen ausführlichen wissenschaftstheoretischen Teil mit einer elementaren Einführung in logische Verfahren. Wagschal nimmt besonders auf Beispiele aus der vergleichenden Forschung Bezug. ${ }^{26}$

Schließlich stellt in Begutachtungsprozessen die theoretisch angeleitete professionelle Methodenanwendung mittlerweile eines der entscheidenden Qualitätsmerkmale der politikwissenschaftlichen Forschung dar, sei es für die Bewilligung von Drittmitteln, sei es für die Publikation von Zeitschriftenaufsätzen. Kittel hat in einer Inhaltsanalyse vier politikwissenschaftlicher Zeitschriften über 40 Jahre herausgefunden, ${ }^{27}$ dass namentlich in der PVS der Anteil der 'theoriegeleitet-empirischen' Artikel von 22,5\% in den 1970er-Jahren auf über 57\% seit 2000 gestiegen ist. Zwar ist der Anstieg in den anderen untersuchten Zeitschriften weit weniger deutlich, der allgemeine Trend wird jedoch untermauert. In den USA wird die professionelle empirische Analyse noch unbedingter als conditio sine qua non für die Zeitschriftenpublikation verlangt (vgl. die Diskussion in Abschnitt 5). Und da sich auch die deutschen Politikwissenschaftlerinnen und Politikwissenschaftler zunehmend international und insbesondere am angelsächsischen Markt orientieren, leistet dieses formale Leistungskriterium der methodischen Professionalisierung weiter Vorschub.

\section{Die "Quali-Quanti-Debatte" als dominante Kontroverse}

Wie eingangs bereits kurz angesprochen, ist die wohl bedeutendste und weitreichendste Kontroverse in den empirischen Sozialwissenschaften diejenige, die häufig mit der Gegenüberstellung 'Quantitativ' vs. 'Qualitativ' verbunden wird. Auch wenn die Wahl dieser Begriffe aus mehreren triftigen Gründen als unglücklich bezeichnet werden muss, so wollen wir im Folgenden an ihr festhalten, weil sich die entsprechenden Kennzeichnungen der Lager nun einmal so durchgesetzt haben. Die beiden Forschungsparadigmata zeichnen sich dadurch aus, dass sie auf einer Reihe methodologischer und erkenntnistheoretischer Vorentscheidung jeweils unterschiedliche Schwerpunktsetzungen vornehmen. So wird

24 Uwe Gehring/Cornelia Weins, Grundkurs Statistik für Politologen und Soziologen, Wiesbaden 2009.

25 Joachim Behnke/Nathalie Behnke, Grundlagen der statistischen Datenanalyse. Eine Einführung für Politikwissenschaftler, Wiesbaden 2006.

26 Uwe Wagschal, Statistik für Politikwissenschaftler, München 1999.

27 B. Kittel (Anm. 2), S. $580 \mathrm{ff}$. die quantitative Forschung bspw. dadurch charakterisiert, dass sie das Modell eines linearen Forschungsprozesses postuliert, dass sie überwiegend deduktiv und hypothesentestend arbeitet, dass sie die Welt in messbare Variablen zerleg und Kausalität anhand der Korrelation von Variablen untersucht ${ }^{28}$, wobei das zugrundeliegende Kausalitätsmodell auf dem Konzept kontrafaktischer Aussagen basiert. ${ }^{29}$ Demgegenüber vertritt das qualitative Paradigma die Notwendigkeit eines zirkulären Forschungsprozesses, einer induktiven und theoriebildenden Forschung, die analytische und interpretative Offenheit gegenüber dem Forschungsgegenstand als Ganzem und die rekonstruierend-verstehende Suche nach Kausalität.

An dieser ausschnittsweisen Auflistung von Gegensätzen wird schon deutlich, dass es sich offensichtlich bei den beiden Paradigmata um Idealtypen im Weber'schen Sinne handelt, die in Reinform in der empirischen Forschung kaum auftreten. Umso erstaunlicher ist es, dass in der Vergangenheit die wechselseitige Abgrenzung von den Vertretern der jeweiligen Paradigmata in den meisten Fällen als ideologischer Grabenkampf verstanden wurde, durchaus mit der Betonung auf dem Wörtchen „Kampf“. Dabei traten die beiden Gruppen oft eher als Jünger einer Art von Offenbarung auf denn als rationale Wissenschaftler, die sich aus objektiv nachvollziehbaren Gründen für eine methodische Vorgehensweise entschieden haben. ,... while this debate is not in any sense about religion, its dynamics are best understood as though it were about religion" ${ }^{30}$ Auch wenn andere Autoren harmloser von "zwei Kulturen" sprechen wie Mahoney und Goertz, ${ }^{31}$ so gehen auch sie davon aus, dass diese beiden Kulturen jeweils über eigene ,values, beliefs and norms“" verfügen, ${ }^{32}$ die dem gegenseitigen Verständnis oft im Wege stehen. Dabei wird die Situation auch nicht leichter dadurch, dass die „Bibel“ der Anhänger des quantitativen Lagers, „Designing Social Inquiry“, von imperialistische Zügen nicht immer ganz frei ist und die selbstbewusst-lässige Art der Autoren dieses Buchs, bestimmte erkenntnistheoretische Probleme dadurch zu lösen, dass sie sie schlicht ignorieren, hat nicht wenige Wissenschaftler irritiert, die nicht grundsätzlich die Welt als aus Nägeln bestehend wahrnehmen wollen, nur weil sie über den entsprechenden (statistischen) Hammer verfügen. Während quantitative Forscher gern der qualitativen Forschung die Wissenschaftlichkeit absprechen wollen, weil sie nicht objektiv ge-

28 Für eine dem quantitativen Paradigma gegenüber kritisch eingestellte Liste relevanter Kriterien für die beiden Paradigmata vgl. bspw. Lamnek (1995, Kap. 2.1 und 2.2).

29 Gary King/Robert O. Keohane/Sidney Verba, Designing Social Inquiry, Princeton, New Jersey 1994.

30 Philip A. Schrodt, Beyond the Linear Frequentist Orthodoxy, in: Political Analysis, 14 (2006), S. 335-339, S. 335

31 James Mahoney/Gary Goertz, A Tale of Two Cultures: Contrasting Quantitative and Qualitative Research, in: Political Analysis, 14 (2006), S. 227-249.

32 Ebd., S. 227 
nug sei, zu interpretativ und damit letztlich beliebig, sehen sich statistisch und empirische arbeitende Sozialwissenschaftlicher ihrerseits von Seiten des qualitativen Lagers nicht selten dem Generalverdacht ausgesetzt, dass die mangelnde Berücksichtigung der Komplexität der Welt in ihren Modellierungen letztlich nur den Mängeln an kognitiver Komplexität der statistisch orientierten Forscher selbst geschuldet ist, die sie zu einem unreflektierten Dasein als positivistische Erbsenzähler verdammen.

Auch institutionell hat sich die "Quali-Quanti-Debatte“ in den beiden großen Vereinigungen DGS und APSA niedergeschlagen. In der APSA gründete sich 2002 die "Qualitative and Multi-Method Research Section“, die sich damit ein eigenes Zuhause schaffen wollten, das sie in der quantitativ dominierten Sektion „Political Methodology“ nicht zu finden glaubte. Eine ganz analoge Entwicklung fand bei der DGS statt, wo sich ebenfalls nach teilweise heftig geführten Auseinandersetzungen in der Sektion "Methoden der empirischen Sozialforschung 2003 die Sektion „Methoden der qualitativen Sozialforschung“ bildete. Im Gegensatz hierzu hat sich dieser Grabenkampf in der DVPW nicht institutionell ausgewirkt. Im Gründungsdokument der Ad-hoc Gruppe "Methoden" der DVPW wurde sogar ausdrücklich das Anliegen betont, beide Traditionen gemeinsam zu berücksichtigen. ${ }^{33}$ Zwar wurde der überwiegend ideologische Krieg zwischen den qualitativ und den quantitativ orientierten Forschern teilweise mit großer Verbissenheit geführt, es zeichnet sich aber doch seit einigen Jahren die Einsich ab, dass keines der beiden Paradigmata einen Heilsanspruch erheben kann. Die wechselseitige Akzeptanz und das Bewusstsein, dass das jeweils andere Paradigma durchaus auch Schwächen der eigenen Forschung ergänzen und korrigieren kann, nehmen sichtlich zu. Dies äußert sich sowohl in einer steigenden Zahl an Publikationen, die sich um eine integrative Methodenperspektive bemühen, als auch in kleineren und größeren Forschungsprojekten, die explizit auf eine Kombination oder Mischformen zwischen qualitativen und quantitativen Vorgehensweisen setzen.

Wie bereits erwähnt bemüht sich bspw. der DVPW-Arbeitkreis Methoden seit seiner Gründung um eine Integration beider Sichtweisen. Dementsprechend wurden in Publikationen des Arbeitskreises ${ }^{34}$ Beiträge aus beiden Traditionen ausgewogen berücksichtigt. Diese Form der integrativen Darstellung von $\mathrm{Me}-$ thoden in Forschung und Lehre ist auch bei anderen neueren Lehrbüchern vorzufinden, so im deutschsprachigen Raum z.B. bei Schmitz und Schubert, Kropp

33 (http://www.dvpw-akmethoden.uni-hamburg.de/meth/kurzb.html).

34 J. Behnke/T. Gschwend/D. Schindler/K.-U. Schnapp (Anm. 19); K.-U. Schnapp N. Behnke/J. Behnke (Anm. 18) und Minkenberg und Pickel et al. ${ }^{35}$ Ähnlich vermittelt im angelsächsischen Raum „Social Science Methodology“" von John Gerring ein „,critical framework“ für jede Form wissenschaftlicher Herangehensweise. ${ }^{36}$ Moses und Knutsen vertreten in „Ways of Knowing“ eine pluralistische Sichtweise auf Methoden. ${ }^{37}$ Um Integration bemüht hat sich auch die Zeitschrift „Political Analysis“, die eigentlich der quantitativ orientierten Methodensektion der APSA zugeordnet ist, indem sie ein Sonderheft zu der Quanti-Quali-Debatte herausgegeben (2006: Jun Ausgabe) hat. In diesem waren unter anderem auch Beiträge zu einem Symposium zu "Rethinking Social Inquiry“ von Brady und Collier enthalten, das die teilweise fundamentale Kritik an „Designing Social Inquiry“ zum Ausdruck bringt. Sehr weit gehen die Bemühungen um eine Integration der beiden Kulturen von den Herausgebern des "Oxford Handbook of Political Methodology“, Janet M. Box-Steffensmeier, Henry Brady und David Collier, ${ }^{38}$ die in ihrem Handbuch eine fein austarierte Mischung von Beiträgen aus beiden Richtungen zusammengestellt haben.

In konkreten Forschungsprojekten findet das Bemühen um Integration Anwendung unter dem Label „Multi-Method“ oder „Mixed Methods“. Methodisch reflektierte Umsetzungen finden sich bspw. bei Heichel und Sommerer. ${ }^{39}$ Mi den komplementären Ergänzungen in so genannter ,nested analysis“ setzt sich Rohlfing kritisch auseinander, ${ }^{40}$ der einen Kriterienkatalog entwickelt, anhand dessen die Angemessenheit des Einsatzes bestimmter Methoden beurteilt werden soll. Aus unserer Sicht bleibt zu hoffen, dass sich diese integrativen Tendenzen zum Nutzen der Qualität und Realitätsnähe empirischer Forschung fortsetzen.

35 Sven-Uwe Schmitz/Klaus Schubert (Hrsg.), Einführung in die Politische Theorie und Methodenlehre, Opladen 2006; S. Kropp/ M. Minkenberg (Anm. 21); S. Pickel/G. Pickel/H.-J. Lauth/D. Jahn (Anm. 22).

36 John Gerring, Social Science Methodology. A Critical Framework, Cambridge 2001.

37 Jonathon W. Moses/Torbjorn L. Knutsen, Ways of Knowing. Competing Methodologies in Social and Political Research, New York, N.Y. 2007.

38 Henry E. Brady/David Collier (Hrsg.), Rethinking Social Inquiry: Diverse Tools, Shared Standards, Lanham 2004.

39 Stefan Heichel/Thomas Sommerer, Mixed-Methodologies: Ein kombiniertes quantitatives und qualitatives Forschungsdesign in der Policy-Analyse am Beispiel eines Projekts zu Konvergenz in der Umweltpolitik, in: Kai-Uwe Schnapp/Nathalie Behnke/Joachim Behnke (Hrsg.), Datenwelten. Datenerhebung und Datenbestände in der Politikwissenschaft, Baden-Baden 2009, S. 200-217.

40 Ingo Rohlfing, What You See and What You Get: Pitfalls and Principles of Nested Analysis in Comparative Research, in: Comparative Political Studies, 41 (2008), S. 1492 1514. 
4 Neue Schwerpunkte

Eine der interessantesten Entwicklungen der letzten Jahre ist, dass die Umfrageforschung etwas von ihrer dominanten Rolle in der politikwissenschaftlichen Forschung zu verlieren scheint und Experimente (Faas 2009) und Simulationen (Martin/Plümper 2006; Shikano 2008; Behnke 2009) sich in den letzten Jahren wachsender Beliebtheit erfreuen konnten. ${ }^{41}$ Dies hängt unter anderem wohl damit zusammen, dass die Entwicklung der Bedeutung dieser beiden Verfahren mit anderen Strömungen oder Entwicklungstendenzen zusammentrifft, so dass sich entsprechende Anknüpfungspunkte ergeben. So spielen Experimente einerseits eine Schlüsselrolle in der sogenannten EITM-Bewegung (Empirical Implication of theoretical Models), die sich für die Entwicklung theoretischer formaler Modelle ausspricht, die dann einer empirischen Überprüfung ausgesetzt werden können. ${ }^{42}$ Die EITM-Bewegung hat auch hierzulande ihre Anhänger gefunden, als Beleg sei dabei nur der Umstand genannt, dass im Sommer 2010 die zweite EITM-Summer School an der Universität Mannheim ausgerichtet wird. Gleichzeitig treten aber bei Experimenten die Fragen des Designs einer Untersuchung besonders in den Fokus, was wiederum eine gewisse Nähe zu vergleichend arbeitenden Politologen schaff. ${ }^{43}$

Die gewachsene Popularität von Simulationen verdankt sich zum Einen wiederum dem EITM-Gedanken, da z.B. so genannte Agentenbasierte Modelle besonders geeignet sind, formale Modelle mit Anleihen aus der Spieltheorie in quasi-empirischen Simulationen zu überprüfen. ${ }^{44}$ Zum Zweiten aber hat die Simulationstechnik an Bedeutung gewonnen, weil bei bayesianischen Verfahren Simulationen zur Schätzung von Konfidenzintervallen eingesetzt werden und bayesianische Schätzverfahren derzeit einen starken neuen Trend bei statistischen Verfahren darstellen. ${ }^{45}$ In der Tat eröffnen bayesianische Simulationen häufig auch ganz neue Möglichkeiten, die mit dem herkömmlichen frequentist-

41 Thorsten Faas, Das Experiment - ein unbekanntes Wesen?, in: Kai-Uwe Schnapp Nathalie Behnke/Joachim Behnke (Hrsg.), Datenwelten. Datenerhebung und Datenbestände in der Politikwissenschaft, Baden-Baden. 2009, S. 72-93; Christian W. Marstände in der Politikwissenschaft, Baden-Baden. 2009, S. 72-93; Christian W. Mar-
tin/Thomas Plümper, Instabilität von parteipolitischen Programmen: Der Einfluss der Zahl der Parteien, in: Thomas Bräuninger/J. Behnke (Hrsg.), Jahrbuch für Handlungsund Entscheidungstheorie. Band 4. Themenschwerpunkt Wahlen und Parteienwettbewerb, Wiesbaden 2006, S. 133-148; S. Shikano, Die Eigendynamik zur Eindimensionalität des Parteienwettbewerbs: Eine Simulationsstudie, in: Politische Vierteljahresschrift, 49 (2008), S. 229-250; N. Behnke (Anm. 17), S. 392-408.

42 Rebecca B. Morton, Methods and models. A guide to the empirical analysis of formal models in political science, Cambridge 2005.

$43 \mathrm{Vgl}$. T. Gschwend/F. Schimmelfennig (Anm. 20).

44 N. Behnke (Anm. 17), S. 392-408.

45 Andrew Gelman/Jennifer Hill, Data Analysis using Regression and Multilevel/ Hierarchical Models, Cambridge 2007. ischen Ansatz nicht zur Verfügung gestanden hätten. So demonstriert z.B. Koller-Meinefelder, ${ }^{46}$ wie bayesianische Simulation zur Imputation von fehlenden Werten eingesetzt werden kann, die durch das Design bewusst kreiert wurden, um die Belastung von Befragten bei Umfragen nicht zu hoch zu treiben. Interessanterweise scheinen für einige Vertreter des Methodenpluralismus bayesianische Verfahren auch besonders geeignet, eine Art Brückenkopf zwischen qualitativ und quantitativ arbeitenden Forschern zu bilden. ${ }^{47}$

Des Weiteren haben sich auch gewisse Verfahren der Diskursanalyse entwickelt, die sich mit Entscheidungsprozessen beschäftigen, indem sie die Entwicklung der Argumentation im Prozess des Diskurses, des Argumentierens und des Verhandelns untersuchen. Holzinger (2004) greift hierbei auf die Sprechakttheorie von Austin zurïck, Nullmeier et al. entwickeln ein differenziertes inhaltsanalytisches Kategorienschema, um den Prozess des Zustandekommens von Gremienentscheidungen $\mathrm{zu}$ untersuchen. ${ }^{48}$ Auch hier gibt es eine gewisse Nähe zu formalen Theorien, wobei der formale Aspekt hier allerdings nicht in der Theorie über den Untersuchungsgegenstand, sondern interessanterweise in der Datenbildung zum Tragen kommt.

Diesen neueren Entwicklungen ist also allen zumindest mehr oder weniger gemein, dass sie sich in Entwicklungslinien im Sinne von EITM oder Multibzw. Mixed Methods einordnen lassen. Auch wenn das Standardverfahren der empirischen Analyse, statistische lineare Gleichungsmodelle, sicherlich immer noch vorherrschend ist, so lässt sich generell eine größere Offenheit bei der Wahl der Instrumente beobachten. Dabei gewinnen Überlegungen zum angemessenen Design einer Untersuchung immer mehr an Gewicht, üblicherweise vor allem dann, wenn sie in Verbindung mit qualitativen Methoden gebracht werden. Unabhängig von der Ergiebigkeit der Integration qualitativer und quantitativer Methoden lässt sich daher die Vermutung aussprechen, dass die Debatte zumindest zu einer größeren Sensibilität gegenüber Designfragen geführt hat.

Ansonsten lassen sich einige weitere Trends bei Auswertungsverfahren feststellen, die hier nur übersichtsweise angerissen werden können (zur Beschreibung einzelner Verfahren vgl. Behnke et al. 2006 und Box-Steffensmeier et al.

46 Florian Koller-Meinefelder, Fragebogensplitting - Reduzierung der Antwortbelastung durch Datenergänzung, in: Kai-Uwe Schnapp/Nathalie Behnke/Joachim Behnke (Hrsg.), Datenwelten. Datenerhebung und Datenbestände in der Politikwissenschaft, BadenBaden 2009, S. 152-173.

47 J. W. Moses/ T. L. Knutsen (Anm. 36).

48 Vgl. Katharina Holzinger, Bargaining through Arguing: An Empirical Analysis Based on Speech Act Theory, in: Political Communication, 21 (2004), S. 195-222; Frank Nullmeier/Tanja Pritzlaff/Anne C. Weihe/Britta Baumgarten, Entscheiden in Gremien. Von der Videoaufzeichnung zur Prozessanalyse., Wiesbaden 2008. 
2008). ${ }^{49}$ Das für Anhänger der qualitativen Kultur zentrale Konzept des Kontexts hat in Form von Mehrebenenanalysen auch in der herkömmlichen Statistik Einzug gehalten und spielt auch im Rahmen von Netzwerkanalyen, die sich ebenfalls immer größerer Beliebtheit erfreuen, eine bedeutende Rolle. Neben der Weiterentwicklung von Zeitreihen- und Panelanalysen entwickelte sich eine neue Klasse räumlicher Regressionsmodelle, die den ersteren Analyseverfahren dadurch nahestehen, dass sie mit einer ähnlichen methodischen Problemkonstellation konfrontiert sind, wie z.B. korrelierenden Fehlern. In diesem Zusammenhang ist auch zu bemerken, dass die Maximum-Likelihood-Schätzung klassische Verfahren wie OLS oder GLS immer öfter ersetzt, was ebenfalls u. a. mit den höheren Rechnerkapazitäten zu erklären ist. Die klassischen OLS-Regresssionsmodelle sind zudem in der Regel auf Variablen beschränkt, die Intervallskalenniveau besitzen. Die große Popularität von Maximum-Likelihood-Verfahren ist daher auch damit zu erklären, dass mit ihrer Hilfe ebenfalls Regressionsmodelle mit nominal- und ordinalskalierten abhängigen und unabhängigen Variablen berechnet werden können, die sich z.B. in Form von Discrete-Choice-Modellen und Item-Response-Analysen ebenfalls in den letzten beiden Jahrzehnten wesentlich weiterentwickelt haben.

In der Frage des methodisch fundierten Umgangs mit kleineren Fallzahlen wurde die politikwissenschaftliche Methodendiskussion insbesondere durch Entwicklungen in der vergleichenden Forschung bereichert. Während die Reflexion über Methoden der vergleichenden Politikwissenschaft in Deutschland noch eine äußerst junge Entwicklung ist, wurde in den USA hierzu bereits Ende der 1990er-Jahre eine intensive Debatte geführt, die auch zu eine Explosion an einschlägigen Publikationen führte. ${ }^{50}$

Das interessante an dieser Entwicklung ist, dass hier mit am stärksten ein methodisch integrativer Ansatz vorangetrieben wird. Während früher in der vergleichenden Forschung zwei Extrempole des Forschungsdesigns dominierten - die vertiefende Einzelfallstudie einerseits und die statistische Analyse von ländervergleichenden Makrodaten andererseits - versucht man nun, neue (Mittel)Wege zu beschreiten. Nicht zuletzt in diesem Forschungsfeld hat sich die qualitative Forschung als wissenschaftlichen Standards genügende empirische Vorgehensweise emanzipieren können. Im Wesentlichen lassen sich drei Strategien identifizieren, die systematisch das Problem kleiner und mittlerer Fallzahlen, wie es für die vergleichende Forschung typisch ist, angehen: a) eine Rückbesinnung

49 Zur Beschreibung einzelner Verfahren vgl J. Behnke/T. Gschwend/D. Schindler/K.-U. Schnapp (Anm. 19); Janet M. Box-Steffensmeier/Henry E. Brady/David Collier (Hrsg.), The Oxford Handbok Jo Box-Stensmeier/Henry E. B

50 Vgl. den exzellenten kommentierten Literaturüberblick in Mahoney (2007) sowie die Diskussion, die in der Zeitschrift Comparative Political Studies (2007, Vol. 40, Heft 1) geführt wurde. auf das klassische Fallstudiendesign, das nun aber methodisch reflektierter und vergleichend angewendet wird b) die Anwendung von Boole'scher Algebra und Mill'scher Induktionslogik in Form der "Qualitative Comparative Analysis“ (QCA), wie sie von Charles Ragin für so genannte Crisp-Sets und Fuzzy-Sets eingeführt und durch Schneider und Wagemanns und Cronqvist und BergSchlosser weiter entwickelt wurde c) der bewusste Mix aus statistischen Analysen und qualitativen Vorgehensweisen, bspw. um Kausalität zweifelsfrei zu etablieren. $^{51}$

Die Renaissance des Fallstudiendesigns trägt in erster Linie dazu bei, dieses Design aus seiner Aschenputtel-Ecke als nicht eigentlich wissenschaftliches $\mathrm{Ge-}$ schichtenerzählen herauszuholen. Wie Gerring überzeugend darlegt, ${ }^{52}$ ist jeder theoretisch angeleiteten Fallstudie eine vergleichende Perspektive inhärent, da der Fall zum Zwecke der Analyse in kleinere Analyseeinheiten heruntergebrochen wird. Darüber hinaus ist die Fallstudie nach wie vor der einzige Weg, um kausale Wirkmechanismen zu identifizieren, z.B. in Form von ProcessTracing. ${ }^{53}$ Dem aus der quantitativen Forschung häufig geäußerten Vorwurf, Einzelfallstudien oder vergleichende Fallstudien unterlägen einem SelektionsBias und seien deshalb in ihren Aussagen höchst fehleranfällig, wird dadurch begegnet, dass die kleine Fallzahl dafür aufgrund theoretischer Kriterien aus einer kleinen und relativ homogenen Grundgesamtheit ausgewählt wird.

Auch die QCA kann sich überzeugend argumentativ gegen hergebrachte Kritikpunkte aus dem quantitativen Lager verteidigen: Die Kritik an den Induktionsschemata, wie sie John Stuart Mill entwickelt hatte, lässt sich nicht ohne weiteres auf die QCA übertragen, da dort nicht einzelne Variablen als Bedingungen betrachtet werden, sondern deren Kombination. Auch werden die Methoden der Übereinstimmung und der Differenz nicht in erster Linie zur Identifizierung notwendiger oder hinreichender Bedingungen verwendet, sondern zu deren Eliminierung. Das wichtigste Gegenargument besteht aber darin, dass die QCA auf der Suche nach kausalen Erklärungen einer anderen Analyselogik folgt als statistische Verfahren: Die Identifikation von notwendigen und Sets von hinreichenden Bedingungen beschreibt Kausalität nicht als 'durchschnittlichen Effekt einer

51 J. Gerring, What Is a Case Study and What Is It Good for?, in: American Political Science Review, 98 (2004) 2, S. 341-354; Charles C. Ragin, The Comparative Method. Moving Beyond Qualitative and Quantitative Strategies, Berkeley/Los Angeles/London 1987; C. C. Ragin, Fuzzy-Set Social Science, Chicago 2000; Joachim K. Blatter/Frank Janning/Claudius Wagemann, Qualitative Politikanalyse. Eine Einführung in Forschungsansätze und Methoden, Wiesbaden 2007; Lasse Cronqvist/Dirk Berg-Schlosser, MultiValue QCA, in: Benoit Rihoux/Charles Ragin (Hrsg), Configurational Comparative Value QC, in: Bet sality, in: Comparative Political Studies, 41 (2008) 4/5, S. 412-436.

52 J. Gerring (Anm. 50), S. 341-354

53 F. Schimmelfennig, Prozessanalyse, in: J. Behnke/T. Gschwend/D. Schindler/K.-U. Schnapp (Anm. 19), S. 263-271; J. K. Blatter/F. Janning/C. Wagemann (Anm. 10). 
Variable'; ${ }^{54}$ vielmehr sind unterschiedliche Pfade denkbar, in denen einzelne Bedingungen unterschiedliche Rollen spielen (einmal ist es notwendig, dass sie vorhanden sind, um eine hinreichende Bedingung zu bilden, ein anders Mal ist es notwendig dass sie abwesend sind. Die Vorteile dieser Betrachtung liegen zum einen darin, dass die Aufmerksamkeit auf Bedingungsstrukturen gelenkt wird, zum anderen darin, dass die Bedeutung von Variablen immer als kontextabhängig betrachtet wird.

Vergleichende Fallstudien, die auf kleinen N's beruhen, sind sowohl für die Begriffsbildung und die Hypothesengenerierung als auch für den Theorietest mächtige und häufig geeignetere Instrumente als statistische Analysen. Denn der vergleichenden Forschung liegt in der Regel ein theoretischer Zugang zugrunde, der sich aus verschiedenen Ansätzen speist, da für die einschlägigen Fragestellungen Großtheorien nicht taugen. Mit diesem Theorie-Eklektizismus kann am besten umgegangen werden, indem man die Empirie systematisch auf die Formulierung neuer Hypothesen hin überprüft. Über die Analyse abweichender Fälle den strukturierten Vergleich oder die Analyse von Entwicklungspfaden ist es möglich, neue Wirkzusammenhänge zu entdecken, die in einem hypothesentestenden Verfahren verborgen bleiben würden. ${ }^{55}$ Optimal ist in solchen Fällen häufig eine Kombination von statistischen Verfahren, bspw. um abweichende Fälle zu identifizieren oder die Dimensionen für einen strukturierten Vergleich zu bestimmen, mit einer vertiefenden qualitativen Studie. Auch die Verbindung qualitativer Studien mit formaler Modellbildung ist in dieser Hinsicht eine erfolgversprechende Strategie, wie sie bspw. durch die "Analytic Narratives" entwickelt wurde. $^{56}$

Generell lässt sich festhalten, dass die neue Offenheit speziell in der vergleichenden Forschung, über Paradigmengrenzen hinweg zu experimentieren, um mit typischen Problemen beschränkter Fallauswahl, fehlerhafter Inferenz oder mangelnder Signifikanz umzugehen und gehaltvolle Theorien mittlerer Reichweite zu entwickeln, für die methodische Fortentwicklung des Faches ausgesprochen positive Impulse gesetzt hat. Die qualitativen Methoden konnten eine weitergehende Akzeptanz erringen, es wurden wieder grundlegende wissen-

54 J. Mahoney, Qualitative Methodology and Comparative Politics, in: Comparative Political Studies, 40 (2007) 2, S. 122-144. S. 135.

$55 \mathrm{Vgl}$. Alexander George/Andrew Bennett, Case Studies and Theory Development in the Social Sciences, Cambridge, Mass. 2005; Barney G. Glaser/Anselm L. Strauss (Anm. 8); J. Mahoney/Erin Kimball/Kendra L. Koivu, The Logic of Historical Explanation in the Social Sciences, in: Comparative Political Studies, 42 (2009) 1, S. 114-146.

56 Robert Bates/Avner Greif/Margaret Levi/Jean-Laurent Rosenthal/Barry R. Weingast, Analytic Narratives. Princeton 1998. schaftstheoretische Diskussionen - etwa über das Wesen der Kausalität oder die Logik der Fallauswahl - geführt, und die Chancen der wechselseitigen Ergänzung quantitativer und qualitativer Vorgehensweisen breiter ausgelotet.

\section{Bestehende Defizite und Ausblick}

Ganz ohne Zweifel ist das mittlere Niveau, was statistische aber auch qualitative Auswertungsverfahren angeht, in den letzten Jahren deutlich angestiegen. Ein Defizit, soweit es besteht, liegt daher nicht darin begründet, dass die Methoden unterentwickelt sind, sondern vielmehr, dass das Niveau der Theorienentwicklung im besten Fall damit nicht Schritt gehalten, im schlimmsten Fall sich trotz oder sogar gerade wegen der methodischen Verfeinerung eher zurückgebildet hat. Der von Green und Shapiro in „Pathologies of Rational Choice“ geäußerte Vorwurf, ein bedeutender Teil der derzeitigen Forschung sei eher „method driven" und weniger "theory driven“, ist nicht von der Hand zu weisen. ${ }^{57}$ Dies hat sicherlich nicht zuletzt etwas mit Publikationsstrategien zu tun. Offensichtlich hat es sich bei vielen Zeitschriften als erfolgreiche Strategie erwiesen, Untersuchungen einzureichen, die mit neuen oder neueren Verfahren, die gewissermaßen in „Mode“ bzw. „hip“ sind, durchgeführt wurden. Die Akzeptanz von Artikeln hängt zudem oft von gewissen "Standards" der Theorieformulierung und -prüfung ab, die "gute" wissenschaftliche Forschung auf eine bestimmte Weise des Vorgehens verengt, die einen paradigmatischen Status zugeschrieben bekommt. Im März 2005 fand eine engagierte Debatte auf der Mailliste HPOLMETH der Methodensektion der APSA statt, in der es um die Annahmepraxis von AJPS ging, deren Herausgeber die Position formuliert hatten, dass Artikel mit reiner Theorie ohne die empirischen Implikationen derselben aufzuzeigen, nicht einmal mehr in den Reviewprozess eingespeist würden, was mit der allgemeinen Überbelastung von Herausgebern und Gutachtern begründet wurde. Dieser Hinweis soll als nur ausschnittartiger Beleg dafür gelten, dass es einen nicht unbedingt glücklichen Trend dazu gibt, nur nach bestimmten rigorosen statistischen Methoden überprüfbare Aussagen überhaupt noch als wissenschaftlich gelten zu lassen oder - nur wenig großzügiger - nur diese als den essentiellen Kern von Wissenschaft anzusehen. Die logische Konsequenz eines sich in Hinsicht auf Publikationserfolg strategisch ausrichtenden Wissenschaftlers muss also sein, sich auf Theoriebestandteile zu konzentrieren, die lediglich noch als Vehikel fungieren, um anhand arrivierter statistischer Verfahren überprüft werden zu können. Eine Theoriebildung, die aber von vornherein nur noch unter diesem

57 Donald P. Green/Ian Shapiro, Pathologies of Rational Choice Theory. A Critique of Applications in Political Science, New Haven 1994. 
strategischen Aspekt betrieben wird, ist notwendigerweise beschränkt und führt ebenso notwendigerweise in der Konsequenz häufig dazu, dass mit methodischen Kanonen auf theoretische Spatzen geschossen wird. Der methodische „Mangel" mancher empirischer Untersuchung besteht daher weniger in methodischen Defiziten als vielmehr in einem Mangel an methodischer Selbstbeschränkung. Auch hier könnte Ockhams Rasiermesser, im Sinne einer Ermahnung, das Instrumentarium nicht über das Notwendige hinaus auszuweiten, durchaus eine sinnvolle Maxime im Hinterkopf des methodisch arbeitenden Wissenschaftlers sein. Es ist aber klar, dass in einer Organisation von Wissenschaft, in der das Wettbewerbsprinzip eine immer größere Rolle spielt, der Verzicht auf methodische Profilierung, auch wenn diese mitunter stärker ausfällt als im objektiven Sinn notwendig, als Wettbewerbsnachteil empfunden wird. Auch ist die Neigung psychologisch durchaus nachvollziehbar, erworbenes Wissen auch zur Anwendung bringen zu wollen.

Doch auch „Theorie“ ist nicht gleich „Theorie“. Die bloße ,sachkundige Erörterung" mag mitunter einen tieferen Einblick in den Untersuchungsgegenstand geben als die empirischen Arbeiten mit „klarem Theoriebezug “ 58 Gerade Arbeiten, bei denen die „empirischen Bezüge jedoch nur beispielhaft, illustrativ und ohne erkennbare methodisch fundierte Systematik erfolgen" "59 sind oft diejenigen, die andere Forschungsarbeiten in ihrer Rolle als Ideengeber mit am stärksten anstoßen. Dies gilt zum Beispiel für zwei der einflussreichsten Bücher der Politikwissenschaft überhaupt, für Downs” „Economic Theory of Democracy“ und für Ostroms „Governing the Commons“. Downs verweist in punkto Empirie bestenfalls auf Alltagsbeobachtungen, Ostrom wiederum verwendet spieltheoretische Modelle lediglich zur Illustration spezifischer Anreizstrukturen ihrer Fallbeispiele zu Common Pool Ressources.

So erfreulich daher einerseits die wachsende Bedeutung der Methoden im Fach ist, wie sie sich auch an der Einrichtung entsprechender Lehrstühle zeigt, so problematisch ist der gleichzeitig stattfindende Abbau der Politischen Theorie. Empirische Arbeit ohne Theorie ist blind, Theorie ohne jeglichen Bezug zur Empirie hingegen bleibt stumm. Das größte derzeitige Problem der Methodenentwicklung ist daher, dass sie zu wenig in Einklang mit einer Entwicklung der Theorie einhergeht. Hier könnte Abhilfe geschaffen werden, indem der Theorie einerseits wieder mehr Aufmerksamkeit in den Curricula geschenkt wird, andererseits die grundlegenden Fertigkeiten zur Formulierung wissenschaftlicher

B. Kittel (Anm. 2)

59 Ebd., S.582
Theorien, die mitunter auch mit empirischen Methoden überprüft werden können, ebenfalls in stärkerem Maße vermittelt werden. Dies würde vor allem einen Ausbau der Ausbildung in den Bereichen der Wissenschafts- und Erkenntnistheorie und logischer Propädeutika erfordern.

Auswahlbibliografie

Bates, Robert / Greif, Avner / Levi, Margaret / Rosenthal, Jean-Laurent / Weingast, Barry R.: Analytic Narratives. Princeton 1998.

Behnke, Joachim: Simulation, in: Schnapp, Kai-Uwe / Behnke, Nathalie / Behnke, Joachim (Hrsg.): Datenwelten. Datenerhebung und Datenbestände in der Politikwissenschaft, BadenBaden 2009, S. 174-195.

Behnke, Joachim / Baur, Nina / Behnke, Nathalie: Empirische Methoden der Politikwissenschaft, Paderborn 2010

Behnke, Joachim / Behnke, Nathalie: Grundlagen der statistischen Datenanalyse. Eine Einführung für Politikwissenschaftler, Wiesbaden 2006.

Behnke, Joachim / Gschwend, Thomas / Schindler, Delia / Schnapp, Kai-Uwe (Hrsg.): Methoden der Politikwissenschaft. Neuere qualitative und quantitative Analyseverfahren, BadenBaden 2006.

Behnke, Nathalie: Datenarchive: Überblick und Kommentierung, in: Schnapp, Kai-Uwe Behnke, Nathalie / Behnke, Joachim (Hrsg.): Datenwelten. Datenerhebung und Datenbestände in der Politikwissenschaft, Baden-Baden 2009, S. 392-408

Blatter, Joachim K. / Janning, Frank / Wagemann, Claudius: Qualitative Politikanalyse. Eine Einführung in Forschungsansätze und Methoden, Wiesbaden 2007.

Box-Steffensmeier, Janet M. / Brady, Henry E. / Collier, David (Hrsg.): The Oxford Handbook of Political Methodology, Oxford 2008.

Brady, Henry E. / Collier, David (Hrsg.): Rethinking Social Inquiry: Diverse Tools, Shared Standards, Lanham 2004.

Cronqvist, Lasse / Berg-Schlosser, Dirk: Multi-Value QCA, in: Rihoux, Benoit / Ragin, Charles (Hrsg): Configurational Comparative Methods, Thousand Oaks 2009, S. 69-86.

Diekmann, Andreas: Aktuelle Probleme der empirischen Sozialforschung, in: Diekmann, Andreas (Hrsg.): Methoden der Sozialforschung, Wiesbaden 2006, S. 8-32.

Dreier, Volker: Empirische Politikforschung, München 1997.

Faas, Thorsten: Das Experiment - ein unbekanntes Wesen?, in: Schnapp, Kai-Uwe / Behnke, Nathalie / Behnke, Joachim (Hrsg.): Datenwelten. Datenerhebung und Datenbestände in de Politikwissenschaft, Baden-Baden 2009, S. 72-93.

Gehring, Uwe / Weins, Cornelia: Grundkurs Statistik für Politologen und Soziologen, Wiesbaden 2009.

Gelman, Andrew / Hill, Jennifer: Data Analysis using Regression and Multilevel/Hierarchical Models, Cambridge 2007.

George, Alexander / Bennett, Andrew: Case Studies and Theory Development in the Social Sciences, Cambridge, Mass. 2005.

Gerring, John: Social Science Methodology. A Critical Framework, Cambridge 2001.

ders. What Is a Case Study and What Is It Good for?, in: American Political Science Review, 98 (2004) 2, S. 341-354 
Glaser, Barney G. / Strauss, Anselm L.: The Discovery of Grounded Theory, New York, N.Y. 1967.

Green, Donald P. / Shapiro, Ian: Pathologies of Rational Choice Theory. A Critique of Applications in Political Science, New Haven 1994.

Gschwend, Thomas / Schimmelfennig, Frank (Hrsg.): Forschungsdesign in der Politikwissenschaft: Probleme - Strategien - Anwendungen, Frankfurt am Main 2007.

Heichel, Stefan / Sommerer, Thomas: Mixed-Methodologies: Ein kombiniertes quantitatives und qualitatives Forschungsdesign in der Policy-Analyse am Beispiel eines Projekts zu Konvergenz in der Umweltpolitik, in: Schnapp, Kai-Uwe / Behnke, Nathalie / Behnke, Joachim (Hrsg.): Datenwelten. Datenerhebung und Datenbestände in der Politikwissenschaft, Baden-Baden 2009, S. 200-217.

Hollis, Martin: The Philosophy of Social Science, Cambridge 1994.

Holzinger, Katharina: Bargaining through Arguing: An Empirical Analysis Based on Speech Act Theory, in: Political Communication, 21 (2004), S. 195-222.

Jahn, Detlef: Einführung in die vergleichende Politikwissenschaft, Wiesbaden 2006.

King, Gary / Keohane, Robert O./Verba, Sidney: Designing Social Inquiry, Princeton, New Jersey 1994.

Kittel, Bernhard: Eine Disziplin auf der Suche nach Wissenschaftlichkeit: Entwicklung und Stand der Methoden in der deutschen Politikwissenschaft, in: Politische Vierteljahresschrift, 50 (2009), S. 577-603.

Koller-Meinefelder, Florian: Fragebogensplitting - Reduzierung der Antwortbelastung durch Datenergänzung, in: Schnapp, Kai-Uwe / Behnke, Nathalie / Behnke, Joachim (Hrsg.): Datenwelten. Datenerhebung und Datenbestände in der Politikwissenschaft, Baden-Baden 2009, S. 152-173.

Kropp, Sabine / Minkenberg, Michael (Hrsg.): Vergleichen in der Politikwissenschaft, Wiesbaden 2005.

Kuckartz, Udo: Einführung in die computergestützte Analyse qualitativer Daten, Wiesbaden 2009.

Lamnek, Siegfried: Qualitative Sozialforschung - Band 1 Methodologie, Weinheim 1995.

Lauth, Hans-Joachim / Pickel, Gert / Pickel, Susanne (Hrsg.): Methoden der vergleichenden Politikwissenschaft. Eine Einführung, Wiesbaden 2009.

Mahoney, James: Toward a Unified Theory of Causality, in: Comparative Political Studies, 41 (2008) 4/5, S. 412-436.

Mahoney, James: Qualitative Methodology and Comparative Politics, in: Comparative Political Studies, 40 (2007) 2, S. 122-144.

Mahoney, James / Goertz, Gary: A Tale of Two Cultures: Contrasting Quantitative and Qualitative Research, in: Political Analysis, 14 (2006), S. 227-249.

Mahoney, James / Kimball, Erin / Koivu, Kendra L.: The Logic of Historical Explanation in the Social Sciences, in: Comparative Political Studies, 42 (2009) 1, S. 114-146.

Martin, Christian W. / Plümper, Thomas: Instabilität von parteipolitischen Programmen: Der Einfluss der Zahl der Parteien, in: Bräuninger, Thomas / Behnke, Joachim (Hrsg.): Jahrbuch für Handlungs- und Entscheidungstheorie. Band 4. Themenschwerpunkt Wahlen und Parteienwettbewerb, Wiesbaden 2006, S. 133-148.

Morton, Rebecca B.: Methods and models. A guide to the empirical analysis of formal models in political science, Cambridge 2005 .

Moses, Jonathon W. / Knutsen, Torbjorn L.: Ways of Knowing. Competing Methodologies in Social and Political Research, New York, N.Y. 2007.
Nullmeier, Frank / Pritzlaff, Tanja / Weihe, Anne C. / Baumgarten, Britta: Entscheiden in Gremien. Von der Videoaufzeichnung zur Prozessanalyse., Wiesbaden 2008.

Opp, Karl-Dieter: Methodologie der Sozialwissenschaften, Wiesbaden 2005.

Pickel, Susanne / Pickel, Gert / Lauth, Hans-Joachim / Jahn, Detlef (Hrsg): Methoden der vergleichenden Politik- und Sozialwissenschaft. Neue Entwicklungen und Anwendungen, Wiesbaden 2009.

Plümper, Thomas: Publikationstätigkeit und Rezeptionserfolg der deutschen Politikwissenschaft in internationalen Fachzeitschriften, 1990-2002. Eine bibliometrische Analyse der Veröffentlichungsleistung deutscher politikwissenschaftlicher Fachbereiche und Institute, in: Politische Vierteljahresschrift, 44 (2003), S. 529-544.

Ragin, Charles C.: The Comparative Method. Moving Beyond Qualitative and Quantitative Strategies, Berkeley/Los Angeles/London 1987.

ders.: Fuzzy-Set Social Science, Chicago 2000.

Rohlfing, Ingo: What You See and What You Get: Pitfalls and Principles of Nested Analysis in Comparative Research, in: Comparative Political Studies, 41 (2008), S. 1492-1514.

Schimmelfennig, Frank: Prozessanalyse, in: Behnke, Joachim / Gschwend, Thomas / Schindler, Delia / Schnapp, Kai-Uwe (Hrsg.): Methoden der Politikwissenschaft. Neuere qualitative und quantitative Analyseverfahren, Baden-Baden 2006, S. 263-271.

Schmitz, Sven-Uwe / Schubert, Klaus (Hrsg.): Einführung in die Politische Theorie und Methodenlehre, Opladen 2006.

Schnapp, Kai-Uwe / Behnke, Nathalie / Behnke, Joachim (Hrsg.): Datenwelten. Datenerhebung und Datenbestände in der Politikwissenschaft, Baden-Baden 2009.

Schneider, Carsten Q. / Wagemann, Claudius: Qualitative Comparative Analysis (QCA) und Fuzzy Sets, Opladen 2007.

Schrodt, Philip A.: Beyond the Linear Frequentist Orthodoxy, in: Political Analysis, 14 (2006), S. 335-339.

Shikano, Susumu: Die Eigendynamik zur Eindimensionalität des Parteienwettbewerbs: Eine Simulationsstudie, in: Politische Vierteljahresschrift, 49 (2008), S. 229-250.

Shikano, Susumu / Becker, Axel: KOALA: Ein Programm zur Datengenerierung für diskrete Entscheidungsmodelle in der Koalitionsforschung, in: Schnapp, Kai-Uwe / Behnke, Nathalie / Behnke, Joachim (Hrsg): Datenwelten. Datenerhebung und Datenbestände in der Politikwissenschaft, Baden-Baden 2009, S. 284-293.

Wagschal, Uwe: Statistik für Politikwissenschaftler, München 1999.

Westle, Bettina (Hrsg.): Methoden der Politikwissenschaft, Baden-Baden 2009. 